\title{
Comportamiento físico y mecánico de elementos no estructurales a base de concreto elaborado con fibra de cáscara de arroz
}

Physical and mechanical behavior of non-structural elements elaborated of concrete made with rice husk fiber

Paola A. Martínez-Gatica', Stéphane Hans², Mauricio Araya-Rodríguez ${ }^{3}$

Martínez-Gatica, P.A; Hans, S; Araya-Rodríguez, M. Comportamiento físico y mecánico de elementos no estructurales a base de concreto elaborado con fibra de cáscara de arroz. Tecnología en Marcha. Vol. 34, especial. Movilidad Estudiantil. Octubre 2021. Pág 40-61.

doi) https://doi.org/10.18845/tm.v34i5.5915

1 Ingeniería en Construcción. Instituto Tecnológico de Costa Rica. Costa Rica Correo electrónico: leimar.pao@gmail.com (D) https://orcid.org/0000-0003-2362-3975 


\title{
Palabras clave
}

Bio-concreto; bloques no estructurales; cáscara de arroz; materiales para la construcción.

\section{Resumen}

En el presente trabajo de investigación se estudió el comportamiento físico y mecánico de dosificaciones de concreto con fibras naturales utilizadas para la elaboración de elementos tipo bloques no estructurales. Las dosificaciones fueron elaboradas con un ligante tipo cemento Portland y como único agregado la cáscara de arroz sin ningún tipo de tratamiento previo, en este proyecto, a esta mezcla se le denomina bio-concreto. Este proyecto se realizó en la Escuela Nacional de Trabajos Públicos (ENTPE) de la Universidad de Lyon en alianza de investigación con la Escuela de Ingeniería en Construcción del Tecnológico de Costa Rica. Se estudiaron diferentes dosificaciones de bio-concreto en donde se analizó el comportamiento mecánico ante la compresión en especímenes cilíndricos con el fin de elegir dos dosificaciones, una para Costa Rica y una para Francia.

Una vez elegidas las dosificaciones, se procedió a estudiar el comportamiento mecánico a compresión en cilindros, la flexión en prismas, el comportamiento térmico en especímenes de ambas formulaciones, culminando con el comportamiento mecánico a compresión de bloques de 15x20x40 cm (ancho, alto y largo). Los resultados muestran el cumplimiento con la resistencia mínima ante la compresión de las normas vigentes para Costa Rica y Francia, ambas formulaciones permiten además tener un material con alta capacidad de aislamiento térmico y densidades menores a $1500 \mathrm{~kg} / \mathrm{m} 3$. Por lo que el uso de este material no se limita a muros no estructurales sino también podría ser utilizado en techos, pisos y acabados.

\section{Keywords}

Bio-concrete; non-structural blocks; rice husk; building materials.

\begin{abstract}
In this research work, the physical and mechanical behavior of concrete dosages with natural fibers used for the preparation of non-structural block elements were studied. The dosages were made with a Portland cement binder and as the only aggregate the rice husk without any previous treatment, in this project, this mixture is called bio-concrete. This project was carried out at the National School of Public Works (ENTPE) of the University of Lyon in research alliance with the School of Engineering in Construction of The Tecnológico of Costa Rica. Different formulations of bio-concrete were studied where mechanical behavior like compression in cylindrical specimens was analyzed in order to choose two formulations, one for Costa Rica and one for France. Once the formulations were chosen, it proceeded to study the mechanical compression behavior in cylinders, the flexion in prisms, the thermal behavior in specimens of both formulations, culminating with the mechanical behavior of compression and flexion of blocks of $15 \times 20 \times 40 \mathrm{~cm}$ (width, height and large). The results show compliance with the minimum resistance to compression of the regulations in force for Costa Rica and France, both formulations also allow to have a material with high thermal insulation capacity and densities less than $1500 \mathrm{~kg} / \mathrm{m}^{3}$. Therefore, the material is not limited to be used in walls, but could be also used in ceilings, floors and finishes.
\end{abstract}




\section{Introducción}

La construcción es una de las actividades del ser humano más importantes para el desarrollo de la vida cotidiana, el impacto que genera el sector construcción no solo es positivo, sino que, también genera un impacto negativo en el ambiente como lo expone [1], ésta industria es la responsable de contribuir alrededor de un $8 \%$ de las emisiones de $\mathrm{CO}_{2}$ a nivel mundial.

El concreto es el material más utilizado en la construcción y debido a su demanda mundial, es el segundo recurso más consumido solo siendo superado por el agua según [2]. El cemento, el cual es el aglutinante más común en los concretos, [1] exponen que aproximadamente 4000 millones de toneladas de este se producen anualmente en el mundo.

Para la Unión Europea se calcula que la producción de cemento, fue de un 8,49\% de la producción mundial y para la región de Latinoamérica y el Caribe un 4,20\% de acuerdo con los datos presentados en el informe estadístico del 2018 de FICEM [3].

Ciertamente, todo el concreto producido anualmente no se usa en el mismo tipo de infraestructura, pero gran parte se usa en bienes raíces donde se utiliza tanto en elementos estructurales (particularmente en carga) como en elementos no estructurales (relleno). Este último punto es importante y hace posible considerar reemplazar este concreto no estructural con un material más ecológico que quizás sea menos eficiente mecánicamente, pero que cumpla con los requisitos de construcción y que pueda tener funciones adicionales como aislamiento térmico, acústico y regulador ambiental.

En el pasado, diferentes autores han investigado sobre concretos que incluyen fibras naturales ya sea para mejorar el comportamiento mecánico de este material o bien sustituir parcial o totalmente los agregados pétreos convencionales de las mezclas de concreto. Autores como [4] que incluyeron fibras de bagazo de caña de azúcar en diferentes porcentajes como fibra de refuerzo en la mezcla de concreto para mejorar las propiedades mecánicas, como ejemplo donde los agregados tradicionales (piedra y arena) se remplazan en totalidad por una fibra natural como el cáñamo se puede mencionar el trabajo de [5].

Más allegado a este proyecto, se destaca el trabajo realizado por [6] quien en su investigación incluyo en diferentes porcentajes la cáscara de arroz en la mezcla de cemento, arena y agua para la producción de elementos tipo bloques estructurales y determinar su comportamiento mecánico, además [7] quien trabajo la cáscara del arroz como único agregado sustituyendo la arena y la piedra en la mezcla de concreto convencional para determinar las propiedades físicas y mecánicas en diferentes dosificaciones de este tipo de mezcla.

Se le llama bio-concreto a la mezcla en la que su matriz cuenta con un agregado de fibra natural, ya sea como agregado único o parcial en esta. Debido a la naturaleza de la fibra esta puede estar siendo reciclada o bien cultivada con el fin de ser utilizada en este tipo de mezclas, lo que saca ventaja de los agregados convencionales es el hecho que, en el proceso de cultivación las plantas de donde se extraen las fibras son capaces de absorber $\mathrm{CO}_{2}$ lo que ayuda a reducir el impacto negativo que pueda generar la extracción de estas o bien la producción de concretos de este tipo.

Esta investigación, se plantea la realización de bloques no estructurales elaborados con bioconcreto de cáscara de arroz, los cuales cumplan con la normativa vigente y de interés para este tipo de elementos no estructurales. Para esto se vuelve necesario plantearse los siguientes objetivos:

- Describir las principales características físicas de la cáscara de arroz utilizada.

- Proponer dos dosificaciones de bio-concreto para la realización de los bloques no estructurales. 
- Medir el comportamiento mecánico en diferentes especímenes elaborados con las dos dosificaciones escogidas.

- Valorar el comportamiento térmico en especímenes elaborados con las dosificaciones escogidas.

Con esto se lograría proponer bloques no estructurales elaborados con un bio-material relativamente nuevo, que sus principales características mecánicas y físicas estén expuestas y un análisis de costos de los materiales involucrados que den una idea inicial de la viabilidad de producción de estos elementos de interés.

El proyecto de investigación se da bajo la alianza entre la Escuela de Ingeniería en Construcción del Tecnológico de Costa Rica y la Escuela Nacional de Trabajos Públicos (ENTPE) de la Universidad de Lyon, en donde se da paso a la realización de proyectos de investigación que son de interés mutuo entre estas escuelas. Por lo que, se plantea proponer una formulación de bio-concreto de cáscara de arroz para Francia y una para Costa Rica para la elaboración de bloques no estructurales, todo el trabajo experimental de laboratorio fue realizado en las instalaciones del ENTPE.

\section{Materiales}

En el análisis de la resistencia a la compresión de diferentes formulaciones del bio-concreto se utilizaron dos tipos de ligantes:

- OPTIMAT Vicat CEM II/B-LL 32,5 RCE NF: cemento tipo II multipropósito, certificado según norma NF EN 197-1, el cual según ficha técnica del fabricante se puede utilizar durante todo el año, adecuado para trabajos de hormigón en climas cálidos y permite un desencofrado rápido. Su composición química es del al menos 65\% de Clinker, 35\% de otras sustancias calizas con un límite de 10\% de puzolanas naturales o cenizas volantes.

- Cemento Natural Rápido Vicat (Cal Natural): certificado según norma NF P 15- 314, es un cemento natural de fraguado rápido, es creado a partir de la cocción a temperatura moderada de piedra caliza arcillosa de composición regular, que se le da molienda muy fina. Tiene presencia de silicato dicálcico activo, aluminato de calcio rico en alúmina y sulfoaluminato de calcio.

Material granular utilizado como único agregado en las mezclas del bio-concreto:

- Cáscara de arroz: proveniente de la región francesa de Camarga, la caracterización física de este material se realizó durante el presente trabajo de investigación.

\section{Metodología}

Obtención de las principales características físicas de la cáscara de arroz a utilizar en el proyecto

Se obtuvo la granulometría mediante el tamizaje del material según la norma ASTM C136, la capacidad de absorción de agua al sumergir el material al agua en diferentes tiempos de estudio y el dato de densidad aparente no compacta del material granular al verterlo en una probeta de ensayo con volumen conocido y siempre desde una altura constante. 
Elaboración de cilindros con diferentes formulaciones de bio-concreto de cáscara de arroz para ser ensayados a compresión

Para un total de 24 formulaciones estudiadas, se elaboraron 6 cilindros del bio-concreto para cada formulación, con el fin de poder someter 3 cilindros al ensayo de resistencia a la compresión a una edad de 7 días y los otros 3 cilindros a una edad de 28 días. La variación entre estas 24 formulaciones fue la relación agregado/ligante y luego la relación agua/ligante.

Una vez que se obtuvieron los resultados del ensayo de resistencia a la compresión, se decidió cuál de las formulaciones estudiadas era la que cumplía con los requisitos mínimos de resistencia establecidos, una resistencia promedio para tres elementos ensayados de 4,14 MPA indicado en la norma de INTECO "INTE C89-2017 (Bloques de concreto para uso no estructuralRequisitos)" para el caso de Costa Rica y un promedio de resistencia a la compresión de 0,30 MPa indicado en el Protocolo de Prueba para la Medición del Rendimiento Umbral de Hormigones de Cáñamo (este como referencia) para el caso de Francia.

Elaboración de elementos no estructurales a base de las formulaciones de bio-concreto de cáscara de arroz escogidas y ensayos mecánicos sobre estos

Una vez escogidas las dos formulaciones que cumplían con los requisitos mínimos de las

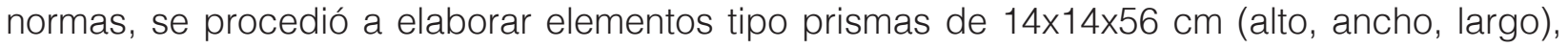
cilindros de 16/32 cm (diámetro/altura), con el fin de realizar ensayos mecánicos de resistencia a compresión a los 7 y 28 días y flexión a los 60 días. Finalmente se realizaron los elementos tipo bloque de $15 \times 20 \times 40 \mathrm{~cm}$ (ancho, alto, largo) y sin agujeros, para ensayarlos ante el ensayo de resistencia a la compresión en bloques según ASTM C1314.

Caracterización física de las formulaciones de bio-concreto escogidas para la elaboración de los bloques no estructurales

Además, se realizaron ensayos físicos sobre muestras extraídas de cilindros elaborados en esta etapa, con el fin de obtener las características higrotérmicas del material. Los ensayos realizados fueron: obtención de la conductividad y efusividad térmica mediante el ensayo del alambre caliente, determinación de propiedades de amortiguación de humedad del material (MBV) según el procedimiento NORDTEST, estudio de las propiedades de transmisión de vapor de agua (WetCup) según norma ISO 12571: 2013.

\section{Resultados}

Con forme a los objetivos propuestos en la investigación y la metodología implementada, se obtuvieron los siguientes resultados.

\section{Caracterización de la cáscara de arroz}

La cáscara de arroz se vertió dentro de una probeta de 2000 ml desde una altura constante de $10 \mathrm{~cm}$, el procedimiento se repitió 6 veces y se promedió el valor de la densidad aparente no compactada obtenida, en el cuadro 1 se presenta el resultado.

Cuadro 1. Densidad no compactada promedio

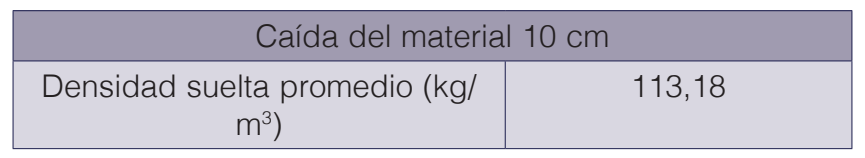

Densidad no compactada promedio a reportar: $\mathrm{\rho}: 113 \mathrm{~kg} / \mathrm{m}^{3}$ 
Se contaba con dos apilamientos del material granular dentro del laboratorio, por lo que se decidió tamizar 200 gramos de cada uno de los apilamientos en 10 grupos de $20 \mathrm{~g}$ para finalmente promediar los resultados para cada apilamiento, el cuadro 2 muestra los promedios obtenidos.

Cuadro 2. Granulometría de la cáscara de arroz.

\begin{tabular}{|c|c|c|c|c|}
\hline \multirow{2}{*}{$\begin{array}{c}\text { Tamiz No. } \\
(\mathrm{mm})\end{array}$} & $\begin{array}{c}|c| \\
\text { Material Retenido en } \\
\text { el tamiz }(\mathrm{g})\end{array}$ & $\begin{array}{c}\text { Ap del material } \\
\text { pasando el tamiz }\end{array}$ & $\begin{array}{c}\text { Material Retenido en } \\
\text { el tamiz }(\mathrm{g})\end{array}$ & $\begin{array}{c}\text { \% del material } \\
\text { pasando el tamiz }\end{array}$ \\
\hline 10,00 & 0,00 & 100,00 & 0,00 & 100,00 \\
\hline 5,00 & 0,00 & 100,00 & 0,00 & 100,00 \\
\hline 2,50 & 0,26 & 98,67 & 1,34 & 98,21 \\
\hline 1,25 & 16,68 & 14,92 & 15,91 & 12,89 \\
\hline 1,00 & 0,63 & 11,75 & 0,73 & 9,18 \\
\hline 0,63 & 1,85 & 2,44 & 1,40 & 2,13 \\
\hline Charola & 0,49 & & 0,42 & \\
\hline$\Sigma$ & 19,91 & & 19,81 & \\
\hline
\end{tabular}

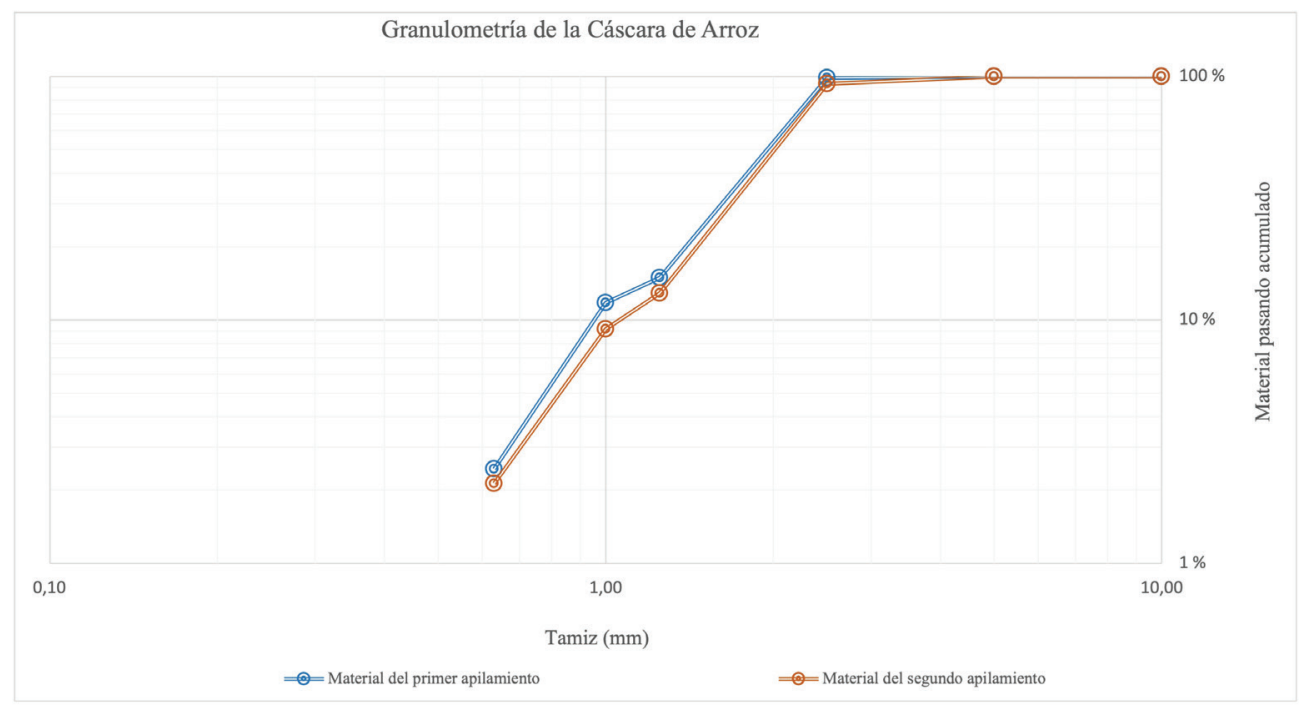

Figura 1. Curva granulométrica de la cáscara de arroz en estudio.

La capacidad de absorción de agua que presentó la cáscara de arroz, se calculó para diferentes tiempos, la ecuación 1 permite obtener el porcentaje de absorción:

$$
\% \text { Absorción }_{B d R}=\left(\frac{M_{H}-M_{S}-X}{M_{B d R}}\right) * 100
$$

(ecuación 1)

Donde:

$\mathrm{M}_{\mathrm{H}}$ : masa húmeda de la bolsa permeable más la cáscara de arroz.

$\mathrm{M}_{\mathrm{S}}$ : masa seca de la bolsa permeable más la cáscara de arroz.

X: cantidad de agua absorbida por la bolsa permeable.

$M_{B d R}$ : masa seca de la cáscara del arroz ingresa en la bolsa permeable. 
En el cuadro 3, se presentan los resultados obtenidos.

Cuadro 3. Información de absorción de la cáscara de arroz en estudio.

\begin{tabular}{|c|c|c|c|}
\hline Tiempo de estudio & $\begin{array}{c}\text { Masa seca promedio } \\
\text { ingresada }(\mathrm{g})\end{array}$ & $\begin{array}{c}\text { Masa húmeda promedio } \\
\text { medida }(\mathrm{g})\end{array}$ & $\begin{array}{c}\% \text { de } \\
\text { absorción }\end{array}$ \\
\hline 0 & - & - & 0 \\
\hline $1 \mathrm{~min}$ & 25,40 & 51,08 & 102,67 \\
\hline $5 \mathrm{~min}$ & 25,13 & 52,29 & 104,01 \\
\hline $15 \mathrm{~min}$ & 25,21 & 52,46 & 108,96 \\
\hline $30 \mathrm{~min}$ & 24,42 & 54,56 & 120,51 \\
\hline $1 \mathrm{~h}$ & 24,33 & 56,45 & 127,61 \\
\hline $6 \mathrm{~h}$ & 24,33 & 58,40 & 135,74 \\
\hline $24 \mathrm{~h}$ & 24,17 & 59,41 & 140,93 \\
\hline $48 \mathrm{~h}$ & 24,23 & 60,72 & 145,90 \\
\hline
\end{tabular}

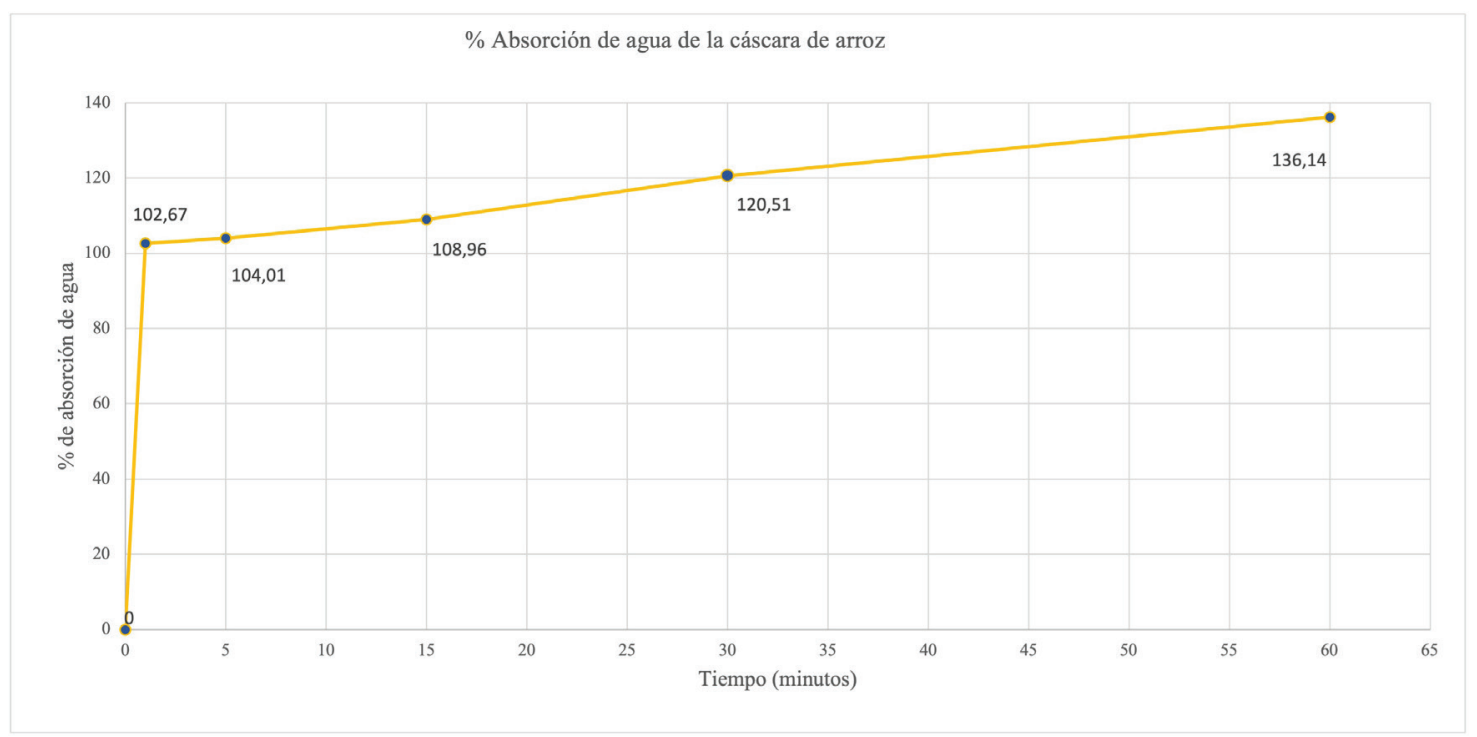

Figura 2. Curva del porcentaje de absorción de agua respecto al tiempo de la cáscara de arroz en estudio.

Ensayo de resistencia a la compresión en cilindros de diferentes formulaciones de bio-concreto de cáscara de arroz

Un total de 24 formulaciones de bio-concreto de cáscara de arroz fueron elaboradas, en donde cada una contó con 6 cilindros elaborados para ser ensayados a compresión y medir su resistencia mecánica ante este tipo de solicitación. Las formulaciones fueron estudiadas en dos fases, en la primera se realizó 6 formulaciones y el ensayo se llevó acabo a los 28 y en la segunda fase se realizaron 18 formulaciones en donde se ensayaron los cilindros a 7 y 28 días de edad. 
Cuadro 4. Resumen de la información principal de las primeras 6 formulaciones estudiadas en la primera fase del proyecto.

\begin{tabular}{|c|c|c|c|c|c|c|}
\hline Formulación & Ligante & $A / L$ & $\begin{array}{c}\rho_{\circ} \\
\left(\mathrm{kg} / \mathrm{m}^{3}\right)\end{array}$ & $\begin{array}{c}\rho_{\mathrm{ae}} \\
\left(\mathrm{kg} / \mathrm{m}^{3}\right)\end{array}$ & $\begin{array}{c}\text { RC. (MPa) } \\
28 \text { días }\end{array}$ & $\begin{array}{l}\mathrm{E}(\mathrm{MPa}) \\
28 \text { días }\end{array}$ \\
\hline F1 F250 & \multirow{3}{*}{$\begin{array}{c}\text { CEM II } 32,5 \\
\text { Optimat } \\
\text { Vicat }\end{array}$} & 0,50 & 668 & 570 & 0,162 & 2,5 \\
\hline F2 F250 & & 0,70 & 766 & 630 & 0,228 & 4,4 \\
\hline F3 F250 & & 0,90 & 826 & 669 & 0,306 & 19,2 \\
\hline F4 F250 & \multirow{3}{*}{ Cal Natural } & 0,70 & 715 & 707 & 0,149 & 1,6 \\
\hline F5 F250 & & 0,90 & 805 & $\left(^{*}\right)$ & - & - \\
\hline F6 F250 & & 1,10 & 832 & 828 & 0,194 & 3,6 \\
\hline
\end{tabular}

Notas sobre cuadro 4.

- Densidad aparente promedio de los cilindros elaborados $\left(\rho_{\circ}\right)$, densidad aparente promedio antes del ensayo de resistencia a la compresión ( $\left.\rho_{\mathrm{ae}}\right)$.

- Resistencia a la compresión máxima promedio de los cilindros ensayados (R.C).

- Módulo de elasticidad aparente promedio.

- El curado de los cilindros consistió en retirarles el molde a los 24 días de edad, en el día 24 ingresarlos al horno por 96 horas a una temperatura constante de $50{ }^{\circ} \mathrm{C}$.

- La formulación F5 no cuenta con información del ensayo de resistencia a la compresión debido a que los cilindros a ensayar se fracturaron durante el traslado del laboratorio hacia los hornos.

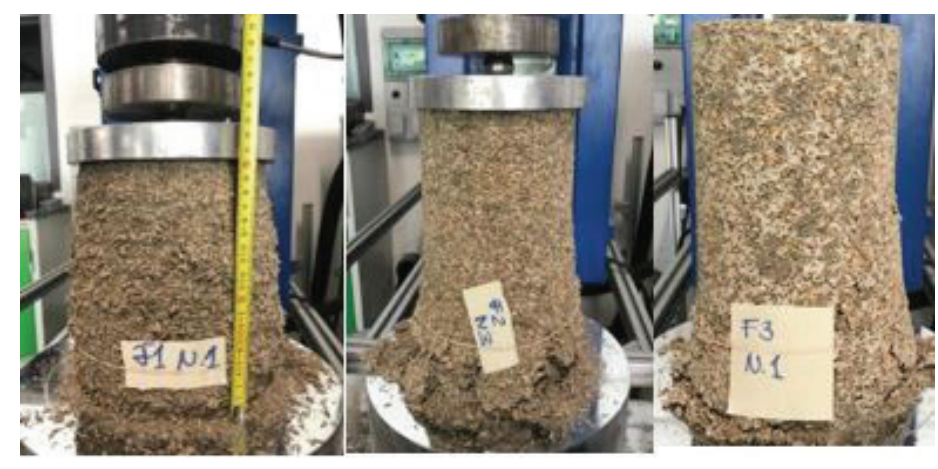

Figura 3. Cilindros de bio-concreto formulaciones F1 a F3 (ligante CEM II $32,5)$ ensayados a la resistencia a la compresión a los 28 días.

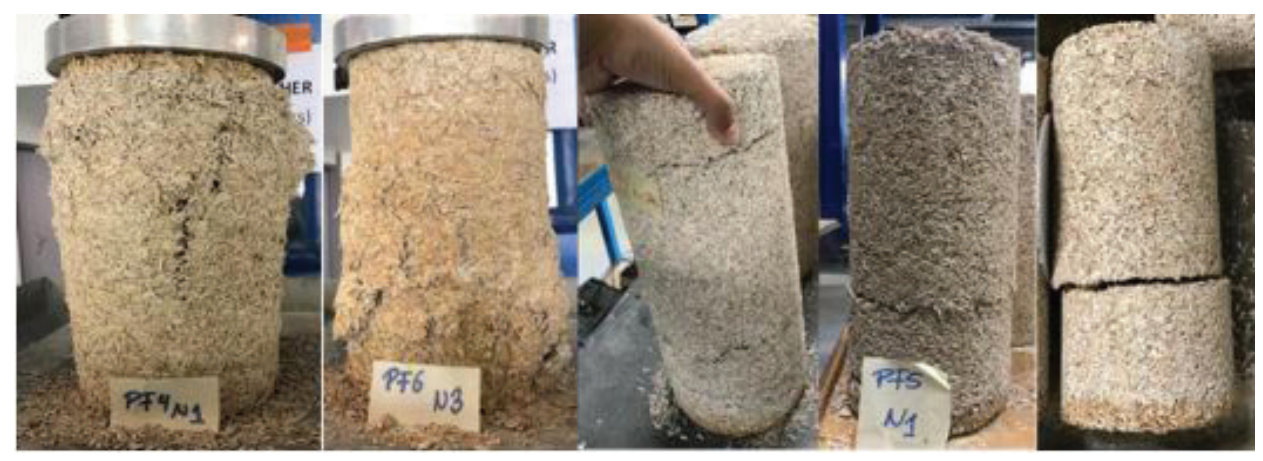

Figura 4. Cilindros de bio-concreto formulaciones F4 y F6 (ligante Cal Natural) ensayados a la resistencia a la compresión a los 28 días y cilindros de formulación F5 (lado izquierdo) fracturados. 
A raíz de estos ensayos, se decidió que la segunda fase de elaboración de cilindros se incluiría el ensayo de resistencia a la compresión de los cilindros a una edad más temprana por lo que se ensayó a los 7 días de edad.

Además, se decide para los cilindros a ensayar a los 28 días, tener un cilindro desmoldado con 1 día de edad y los otros 2 desmoldarlos a los 21 días para ingresarlos al horno por 7 días a una temperatura constante de $50{ }^{\circ} \mathrm{C}$ para tratar de extraer la mayor cantidad de agua libre en los cilindros, esto para las formulaciones de la F8 hasta F20. Para las formulaciones de la F21 hasta F24 los cilindros a ensayar ante compresión a los 28 días de edad igualmente se desmoldaría 1 por formulación con 1 día de edad y los otros 2 en este caso desmoldarlos a los 28 días (mismo día del ensayo), esto con el fin de ver y analizar si los resultados de la resistencia a la compresión cambiaban según tiempo de desmolde e ingreso o no al horno y decidir cuál metodología es la mejor para este tipo de bio-concreto.

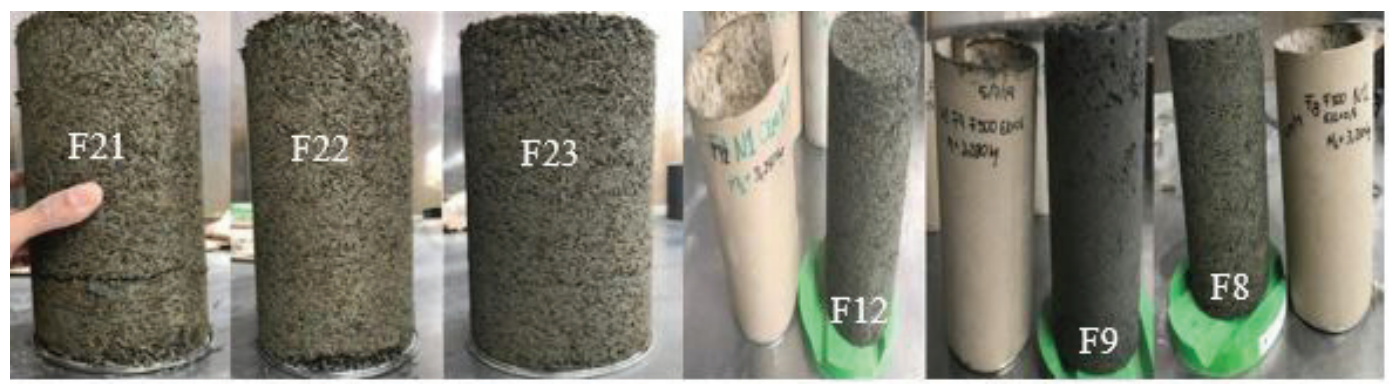

Figura 5. Cilindros de las formulaciones de la segunda fase después de quitar el molde con 24 horas de elaboración.

Cuadro 5. Resumen de la información principal de las formulaciones estudiadas en la segunda fase del proyecto con cilindros ensayados a los 28 días ingresados al horno por 7 días.

\begin{tabular}{|c|c|c|c|c|c|c|c|c|c|}
\hline \multirow{2}{*}{ Formulación } & \multirow{2}{*}{$A / L$} & \multirow{2}{*}{$\begin{array}{c}\rho_{\circ} \\
\left(\mathrm{kg} / \mathrm{m}^{3}\right)\end{array}$} & \multirow{2}{*}{$\begin{array}{c}\text { RC. } \\
(\mathrm{MPa}) \\
7 \text { días }\end{array}$} & \multicolumn{2}{|c|}{$\begin{array}{c}\rho_{\mathrm{ae}} \\
\left(\mathrm{kg} / \mathrm{m}^{3}\right)\end{array}$} & \multicolumn{2}{|c|}{$\begin{array}{c}\text { RC. (MPa) } \\
28 \text { días }\end{array}$} & \multicolumn{2}{|c|}{$\begin{array}{l}\mathrm{E}(\mathrm{MPa}) \\
28 \text { días }\end{array}$} \\
\hline & & & & $\begin{array}{l}\text { Desmolde } \\
\text { a } 1 \text { día }\end{array}$ & $\begin{array}{l}\text { Desmolde } \\
\text { a } 21 \text { días }\end{array}$ & $\begin{array}{l}\text { Desmolde } \\
\text { a } 1 \text { día }\end{array}$ & $\begin{array}{l}\text { Desmolde } \\
\text { a } 21 \text { días }\end{array}$ & $\begin{array}{c}\text { Desmolde } \\
\text { a } 1 \text { día }\end{array}$ & $\begin{array}{l}\text { Desmolde } \\
\text { a } 21 \text { días }\end{array}$ \\
\hline F8 F500 & 0,50 & 1470 & 1,3 & 1149 & 1164 & 1,4 & 1,3 & 140,0 & 136,7 \\
\hline F9 F500 & 0,60 & 1507 & 1,9 & 1112 & 1145 & 2,0 & 2,3 & 175,0 & 195,5 \\
\hline F92 F500 & 0,45 & 1497 & 1,5 & 1167 & 1201 & 1,3 & 1,6 & 112,5 & 130,0 \\
\hline F10 F600 & 0,60 & 1568 & 3,1 & 1149 & 1202 & 3,2 & 3,6 & 242,9 & 297,0 \\
\hline F11 F600 & 0,50 & 1730 & 2,9 & 1373 & 1404 & 3,3 & 3,8 & 229,9 & 370,2 \\
\hline F12 F600 & 0,45 & 1718 & 4,1 & 1408 & 1481 & 3,3 & 4,1 & 236,0 & 402,2 \\
\hline F13 F700 & 0,50 & 1705 & 6,1 & 1388 & 1393 & 6,5 & 6,7 & 555,6 & 615,8 \\
\hline F14 F700 & 0,45 & 1853 & 5,9 & 1477 & 1544 & 6,5 & 6,0 & 512,8 & 641,1 \\
\hline F15 F700 & 0,40 & 1852 & 6,7 & 1527 & 1578 & 7,4 & 6,3 & 563,6 & 604,0 \\
\hline F16 F750 & 0,50 & 1671 & 5,4 & 1324 & 1395 & 5,0 & 6,3 & 512,8 & 673,5 \\
\hline F17 F750 & 0,45 & 1819 & 5,3 & 1479 & 1530 & 6,1 & 5,0 & 520,0 & 615,2 \\
\hline F18 F650 & 0,50 & 1744 & 4,7 & 1362 & 1456 & 4,2 & 4,7 & 483,9 & 531,7 \\
\hline F19 F650 & 0,45 & 1825 & 4,5 & 1440 & 1524 & 4,1 & 4,7 & 433,3 & 515,5 \\
\hline F20 F550 & 0,45 & 1637 & 2,3 & 1256 & 1326 & 2,3 & 2,5 & 133,3 & 197,7 \\
\hline
\end{tabular}


Cuadro 6. Resumen de la información principal de las formulaciones estudiadas en la segunda fase del proyecto con cilindros ensayados a los 28 días sin ingresados al horno.

\begin{tabular}{|c|c|c|c|c|c|c|c|c|c|}
\hline \multirow{2}{*}{ Formulación } & \multirow{2}{*}{$A / L$} & \multirow{2}{*}{$\begin{array}{c}\rho_{\circ} \\
\left(\mathrm{kg} / \mathrm{m}^{3}\right)\end{array}$} & \multirow{2}{*}{$\begin{array}{c}\text { RC. } \\
(\mathrm{MPa}) \\
7 \text { días }\end{array}$} & \multicolumn{2}{|c|}{$\begin{array}{c}\rho_{\mathrm{ae}} \\
\left(\mathrm{kg} / \mathrm{m}^{3}\right)\end{array}$} & \multicolumn{2}{|c|}{$\begin{array}{c}\text { RC. (MPa) } \\
28 \text { días }\end{array}$} & \multicolumn{2}{|c|}{$\begin{array}{l}\text { E (MPa) } \\
28 \text { días }\end{array}$} \\
\hline & & & & $\begin{array}{c}\text { Desmolde } \\
\text { a } 1 \text { día }\end{array}$ & $\begin{array}{l}\text { Desmolde } \\
\text { a } 28 \text { días }\end{array}$ & $\begin{array}{l}\text { Desmolde } \\
\text { a } 1 \text { día }\end{array}$ & $\begin{array}{l}\text { Desmolde } \\
\text { a } 28 \text { días }\end{array}$ & $\begin{array}{c}\text { Desmolde } \\
\text { a } 1 \text { día }\end{array}$ & $\begin{array}{l}\text { Desmolde } \\
\text { a } 28 \text { días }\end{array}$ \\
\hline F21 F280 & 0,50 & 1012 & 0,2 & $\left({ }^{\star}\right)$ & 892 & - & 0,5 & - & 7,5 \\
\hline F22 F300 & 0,50 & 1075 & 0,3 & 807 & 958 & 0,8 & 0,7 & 19,0 & 6,0 \\
\hline F23 F320 & 0,50 & 1137 & 0,3 & 896 & 1018 & 0,7 & 0,7 & 25,0 & 9,5 \\
\hline F24 F350 & 0,50 & 1233 & 0,4 & 979 & 1115 & 1,0 & 1,0 & 68,0 & 40,5 \\
\hline
\end{tabular}

Nota sobre cuadros 5 y 6: el ligante utilizado en todas las formulaciones fue CEM II 32,5 Optimat Vicat.

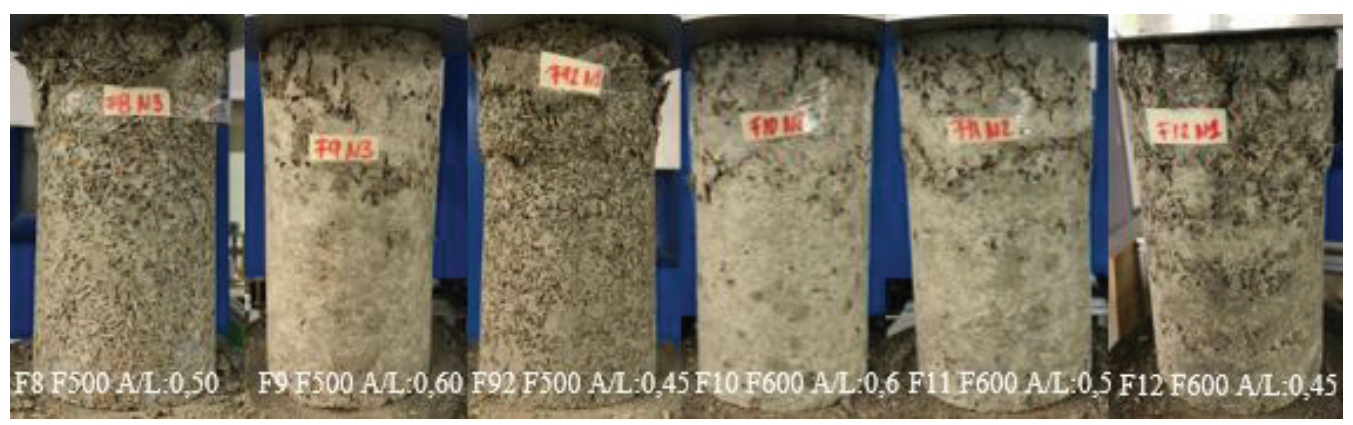

Figura 6. Ejemplo de falla típica presente en los cilindros del grupo de formulaciones F500 y F600.

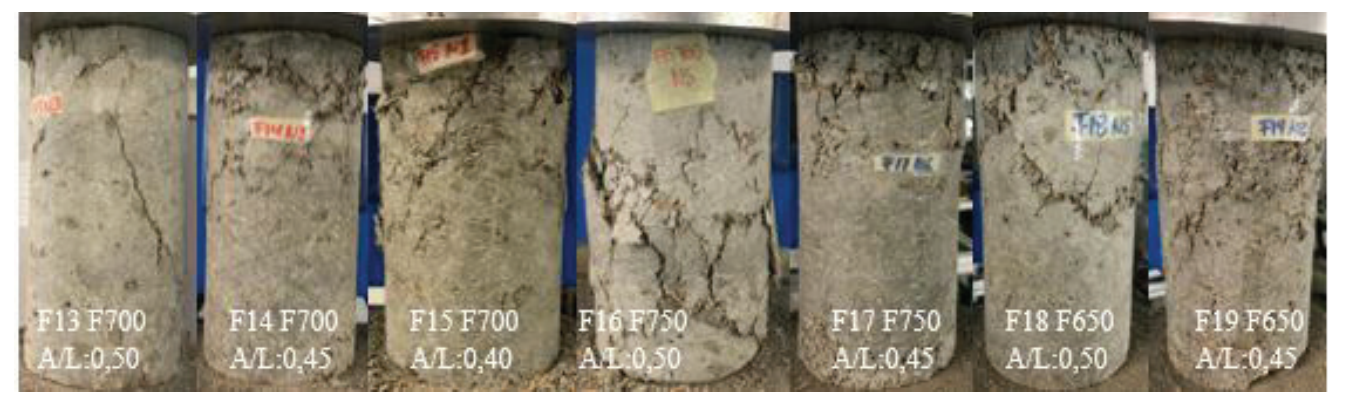

Figura 7. Ejemplo de falla típica presente en los cilindros del grupo de formulaciones F700, F750 y F650.

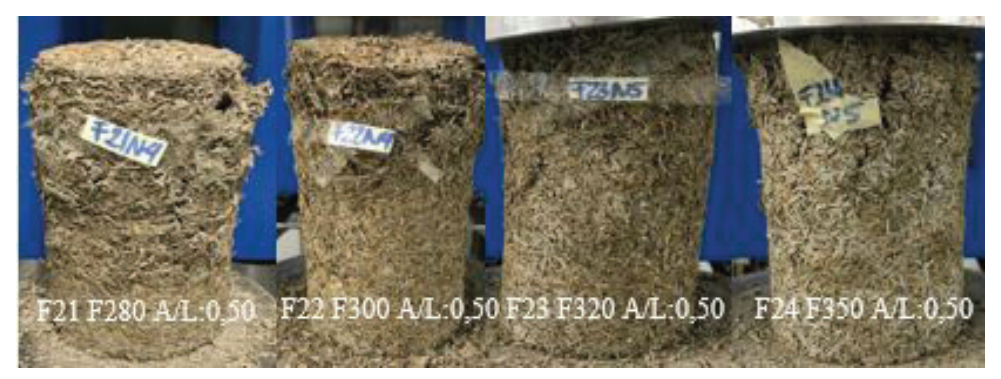

Figura 8. Ejemplo de falla típica presente en los cilindros del grupo de formulaciones F280, F300, F320 y F350. 
Ensayos mecánicos sobre los elementos no estructurales elaborados con las dos formulaciones de bio-concreto de cáscara de arroz escogidas

A partir del estudio de las formulaciones anteriormente presentadas, se pudo escoger basados en la resistencia a la compresión a los 7 días y el módulo de elasticidad aparente la formulación F12 F600 CEM II 32,5 A/L: 0,45 renombrándola FCR para la normativa costarricense y la formulación F22 F300 CEM 32,5 A/L: 0,45 renombrándola FFR para la normativa francesa. La formulación FFR sufrió un cambio en la relación Agua/Ligante pasando de 0,45 a 0,66 por temas de trabajabilidad de la mezcla.

A continuación, se presentan los resultados de resistencia a la compresión sobre cilindros, resistencia a la flexión sobre vigas y resistencia a la compresión sobre bloques no estructurales y sin huecos elaborados con las formulaciones FCR y FFR.

Cuadro 7. Promedio de la información obtenida en el ensayo de compresión a diferentes edades sobre cilindros de las formulaciones FCR y FFR de bio-concreto.

\begin{tabular}{|c|c|c|c|c|c|}
\hline $\begin{array}{l}\text { Edad } \\
\text { (días) }\end{array}$ & $\rho_{0}\left(\mathrm{~kg} / \mathrm{m}^{3}\right)$ & $\begin{array}{l}\text { Fuerza máx. } \\
\qquad(\mathrm{kN})\end{array}$ & $\begin{array}{c}\text { Resistencia a la } \\
\text { compresión máx. } \\
\text { (MPa) }\end{array}$ & $\begin{array}{l}\text { Deformación } \\
\text { para la } R C \text { máx. } \\
(\mathrm{mm} / \mathrm{mm})\end{array}$ & $\begin{array}{l}\text { Módulo de elasticidad } \\
\text { aparente (MPa) }\end{array}$ \\
\hline \multicolumn{6}{|c|}{ Formulación FCR } \\
\hline 7 & - & 68,6 & 3,4 & 0,0136 & 480,4 \\
\hline 28 & 1436 & 96,5 & 4,8 & 0,0166 & 529,9 \\
\hline 77 & 1435 & 110,2 & 5,5 & 0,0154 & 544,5 \\
\hline \multicolumn{6}{|c|}{ Formulación FFR } \\
\hline 7 & - & 7,1 & 0,4 & 0,0838 & 32,5 \\
\hline 28 & 861 & 12,0 & 0,6 & 0,0592 & 61,5 \\
\hline 77 & 869 & 18,0 & 0,9 & 0,0296 & 76,4 \\
\hline
\end{tabular}

Nota sobre el cuadro 7:

- Todos los cilindros fueron desmoldados a los 7 días de edad.

- Ningún cilindro fue ingresado al horno y se dejaron reposar en el laboratorio a temperatura ambiente hasta la fecha de ensayo.

- Se ensayaron tres cilindros por cada edad de ensayo.

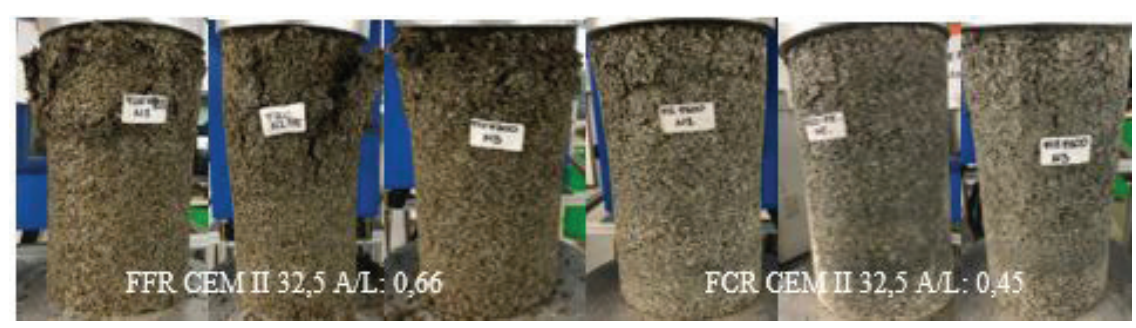

Figura 9. Cilindros de las formulaciones FFR y FCR en la parte superior antes del ensayo de resistencia a la compresión a 7 días y en la parte inferior ya ensayados. 


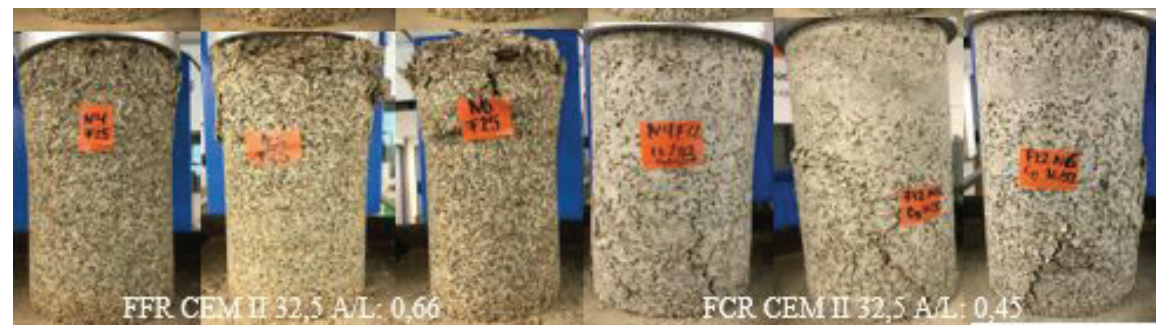

Figura 10. Cilindros de las formulaciones FFR y FCR en la parte superior antes del ensayo de resistencia a la compresión a 28 días y en la parte inferior ya ensayados.

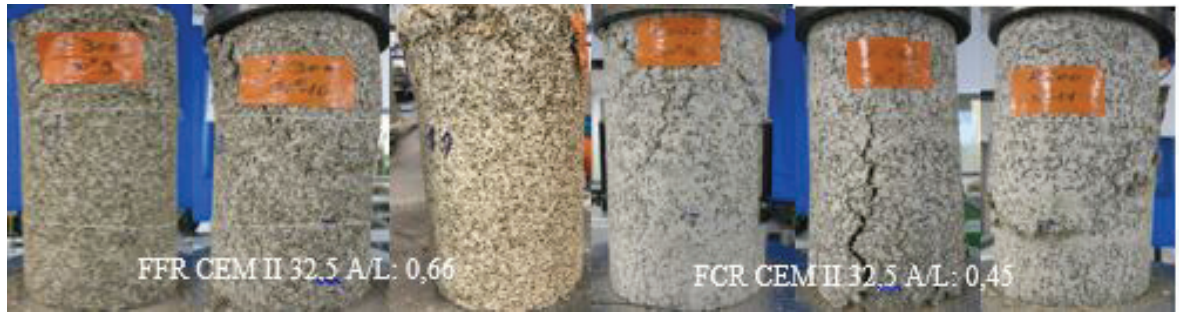

Figura 11. Cilindros de las formulaciones FFR y FCR en la parte superior antes del ensayo de resistencia a la compresión a 77 días y en la parte inferior ya ensayados.

Cuadro 8. Resumen de la información obtenida del ensayo de flexión en 4 puntos sobre prismas de concreto la fibra de cáscara de arroz.

\begin{tabular}{|c|c|c|c|c|c|c|}
\hline Formulación & $\mathrm{A} / \mathrm{L}$ & $\begin{array}{c}\text { Edad del } \\
\text { prisma } \\
(\text { días })\end{array}$ & $\rho_{\mathrm{ae}}\left(\mathrm{kg} / \mathrm{m}^{3}\right)$ & $\begin{array}{c}\text { Fuerza } \\
\text { máx. } \\
(\mathrm{kN})\end{array}$ & $\begin{array}{c}\text { Resistencia a } \\
\text { la flexión } \mathrm{F}_{\mathrm{ct}} \\
\text { máx. }(\mathrm{MPa})\end{array}$ & $\begin{array}{c}\text { Desplazamiento para } \\
\mathrm{F}_{\mathrm{ct}} \text { máx. (mm) }\end{array}$ \\
\hline FCR & 0,45 & 70 & 1509 & 17,97 & 2,75 & 2,56 \\
\hline FFR & 0,66 & 54 & 855 & 6,00 & 0,75 & 3,47 \\
\hline
\end{tabular}

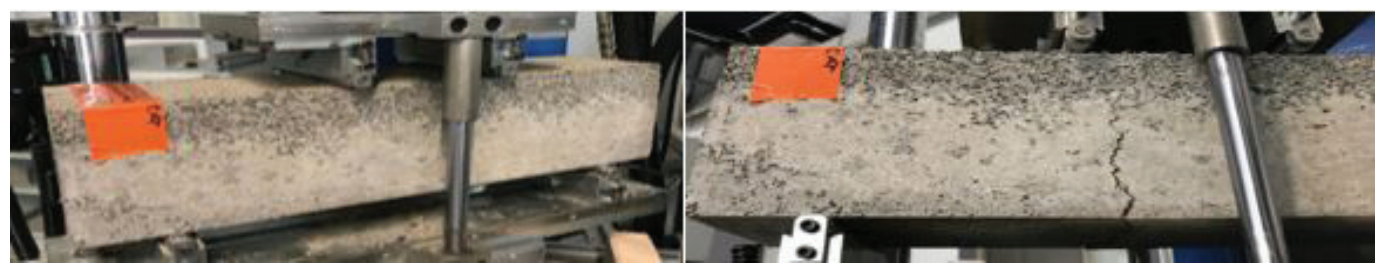

Figura 12. Prisma de bio-concreto formulación FCR (F600 CEM II 32,5 A/L: 0,45) lado izquierdo antes del ensayo de flexión lado derecho después del ensayo de flexión.

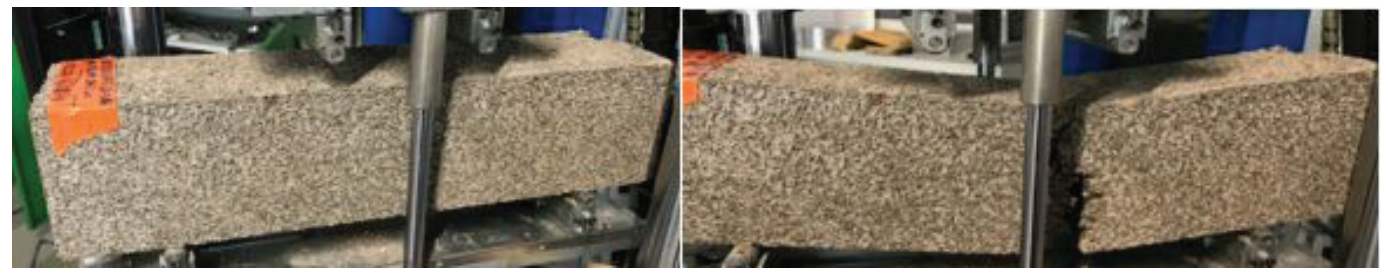

Figura 13. Prisma de bio-concreto formulación FFR (F300 CEM II 32,5 A/L: 0,66) lado izquierdo antes del ensayo de flexión lado derecho después del ensayo de flexión. 
Cuadro 9. Resumen de la información obtenida del ensayo de compresión sobre bloques de bio-concreto (FCR F600 CEM II 32,5 A/L: 0,45).

\begin{tabular}{|c|c|c|c|c|c|}
\hline \multicolumn{6}{|c|}{78 días (jours) } \\
\hline Bloque & pae $\left(\mathrm{kg} / \mathrm{m}^{3}\right)$ & Fuerza máx. & $\begin{array}{c}\text { Resistencia a la } \\
\text { compresión máx. } \\
\text { (MPa) }\end{array}$ & $\varepsilon$ para el $\sigma_{\operatorname{Rmax}}$ & $\begin{array}{c}\text { Resistencia a la } \\
\text { compresión corregida } \\
(\mathrm{MPa})\end{array}$ \\
\hline B1 & 1546 & 594,6 & 9,9 & 0,0463 & 7,4 \\
\hline B2 & 1535 & 648,3 & 10,8 & 0,0397 & 8,1 \\
\hline B3 & 1542 & 623,8 & 10,4 & 0,0456 & 7,8 \\
\hline Promedio & 1541 & 622,2 & 10,4 & 0,0438 & 7,8 \\
\hline
\end{tabular}

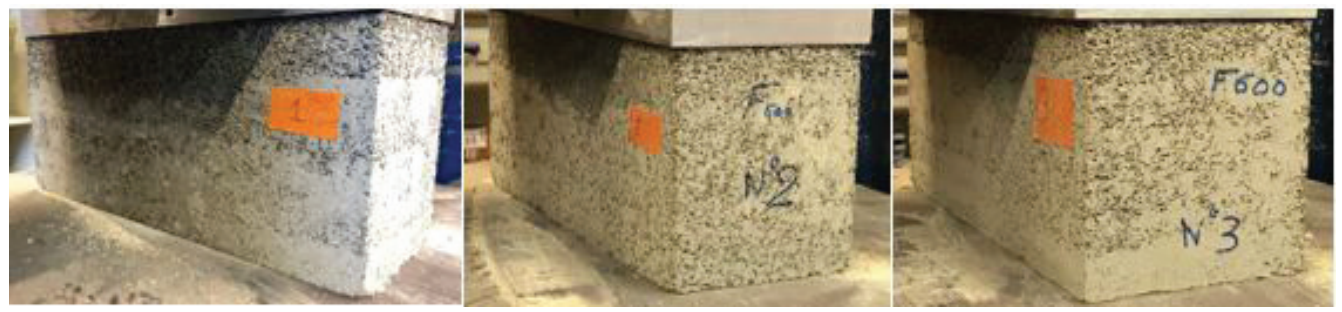

Figura 14. Bloques 1, 2 y 3 de la formulación FCR F600 CEM II 32,5 A/L: 0,45 antes del ensayo de compresión.

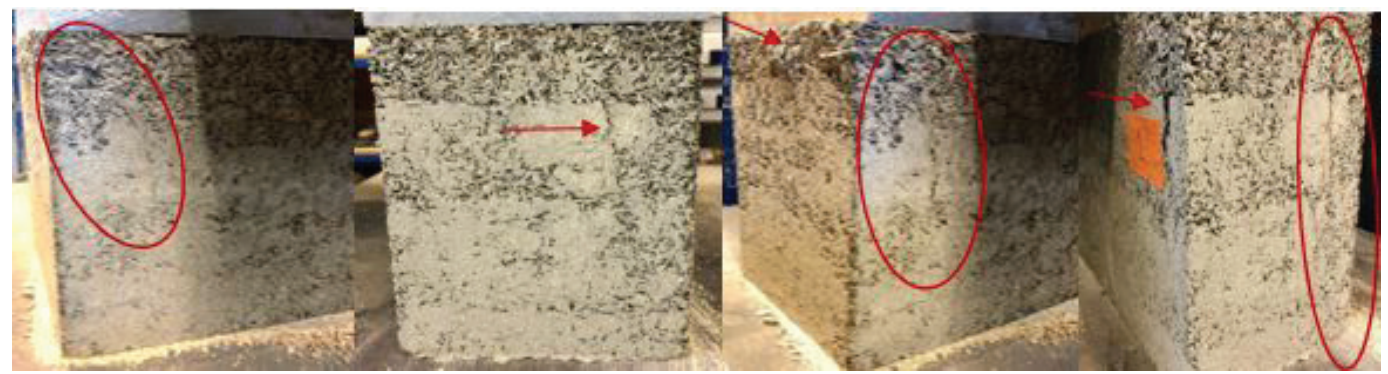

Figura 15. Bloque 1 de la formulación FCR F600 CEM II 32,5 A/L: 0,45 después del ensayo de compresión y las grietas visibles por la falla del espécimen.

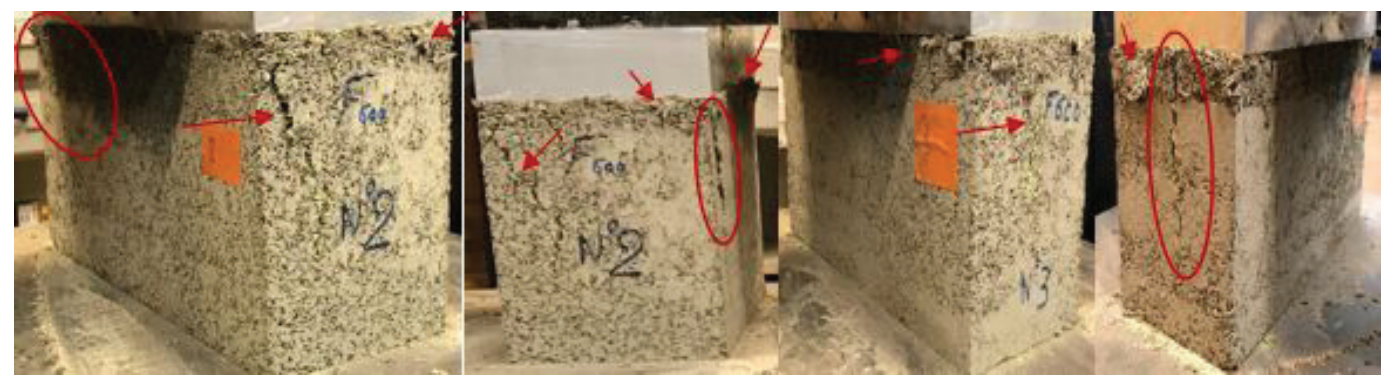

Figura 16. Bloque 2 y 3 de la formulación FCR F600 CEM II 32,5 A/L: 0,45 después del ensayo de compresión y las grietas visibles por la falla del espécimen. 
Cuadro 10. Resumen de la información obtenida del ensayo de compresión sobre bloques de bio-concreto (FFR F300 CEM II 32,5 A/L: 0,66)

\begin{tabular}{|c|c|c|c|c|c|}
\hline \multicolumn{7}{|c|}{55 días (jours) } \\
\hline Bloque & pae $\left(\mathrm{kg} / \mathrm{m}^{3}\right)$ & Fuerza máx. & $\begin{array}{c}\text { Resistencia a la } \\
\text { compresión máx. } \\
(\mathrm{MPa})\end{array}$ & $\begin{array}{c}\text { Resistencia a la } \\
\text { Bara el } \sigma_{\text {Rmax }}\end{array}$ & $\begin{array}{c}\text { Rompresión corregida } \\
(\mathrm{MPa})\end{array}$ \\
\hline B1 & 976 & 109,5 & 1,8 & 0,1054 & 1,4 \\
\hline B2 & 994 & 128,7 & 2,0 & 0,1029 & 1,5 \\
\hline B3 & 1026 & 92,3 & 2,0 & 0,1033 & 1,5 \\
\hline Promedio & 999 & 110,2 & 2,0 & 0,1039 & 1,5 \\
\hline
\end{tabular}

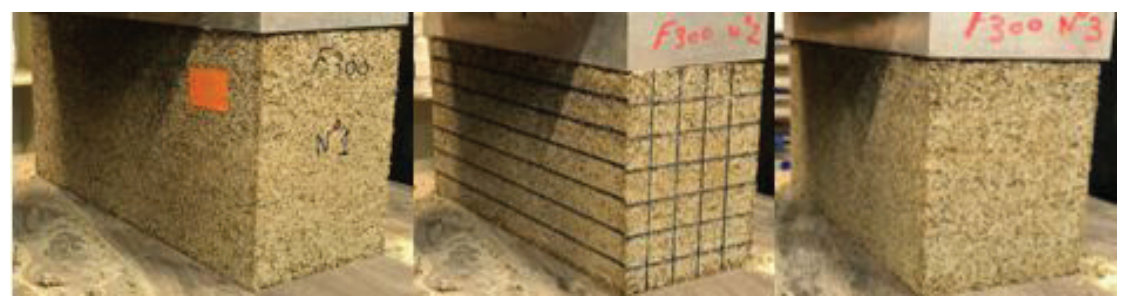

Figura 17. Bloques 1, 2 y 3 de la formulación FFR F300 CEM II 32,5 A/L: 0,66 antes del ensayo de compresión.

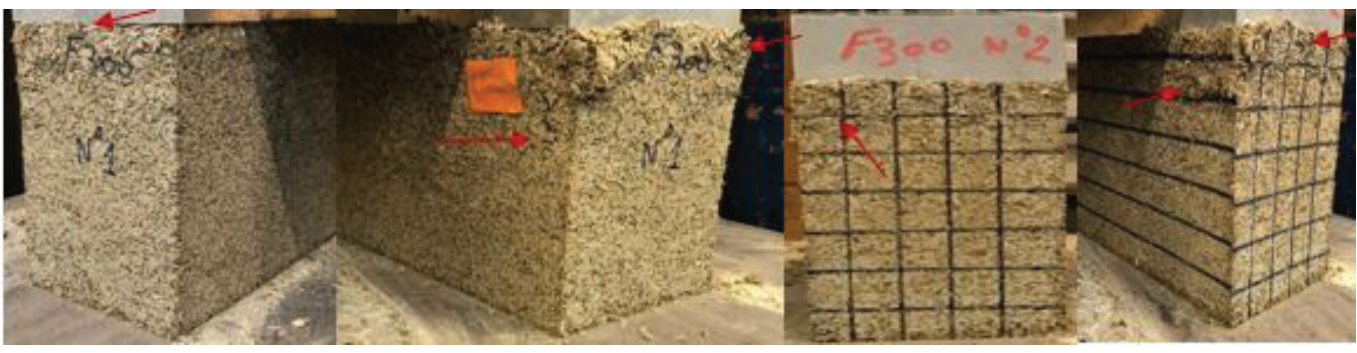

Figura 18. Bloque 1 y 2 de la formulación FFR F300 CEM II 32,5 A/L: 0,66 después del ensayo de compresión y las grietas visibles por la falla del espécimen.

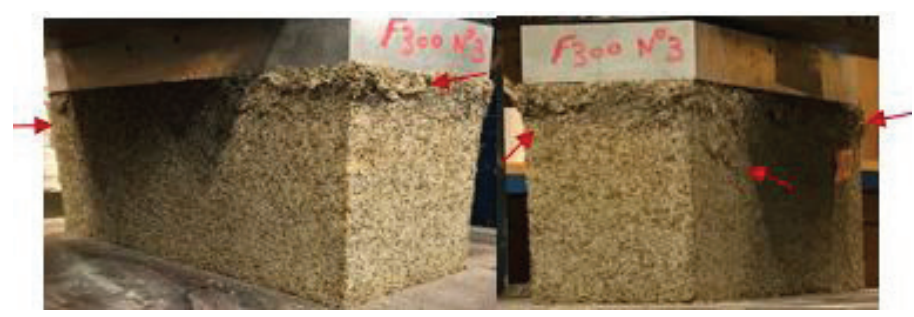

Figura 19. Bloque 3 de la formulación FFR F300 CEM II 32,5 A/L: 0,66 después del ensayo de compresión y las grietas visibles por la falla del espécimen.

Caracterización física de las formulaciones de bio-concreto escogidas para la elaboración de los bloques no estructurales

A continuación, se muestran los resultados del ensayo de Efusividad ( $\beta$ ) y Conductividad $(\lambda)$ Térmica de los especímenes de las formulaciones en estudio. Por otro lado, es importante mencionar que los valores de capacidad calorífica (Cp), difusividad térmica (a) y tiempo de desfase térmico fueron obtenidos de manera indirecta mediante las ecuaciones. 
Cuadro 11. Características térmicas de las formulaciones FFR F300 CEM II 32,5 A/L: 0,66 y FCR F600 CEM II 32,5 A/L: 0,45.

\begin{tabular}{|c|c|c|}
\hline Característica & FFR F300 CEM II 32,5 A/L:0,66 & FCR F600 CEM II 32,5 A/L:0,45 \\
\hline$\rho_{\text {seca }}\left(\mathrm{kg} / \mathrm{m}^{3}\right)$ & 690 & 1240 \\
\hline$\beta\left(\mathrm{J} /\left(\mathrm{K}^{*} \mathrm{~m}^{2 *} \mathrm{~s}^{1 / 2}\right)\right)$ & 322 & 626 \\
\hline $\mathrm{I}\left(\mathrm{W} /\left(\mathrm{m}^{*} \mathrm{~K}\right)\right)$ & 0,125 & 0,259 \\
\hline $\mathrm{Cp}\left(\mathrm{J} /\left(\mathrm{kg}^{*} \mathrm{~K}\right)\right)$ & 1213,05 & 1221,23 \\
\hline$a(\mathrm{~mm} 2 / \mathrm{s})$ & $1,49 \times 10^{-07}$ & $1,71 \times 10^{-07}$ \\
\hline$(\mathrm{h})$ & 8,94 & 8,35 \\
\hline
\end{tabular}

Los resultados del ensayo de MBV, se presentan en la figura 20 y cuadro 12.

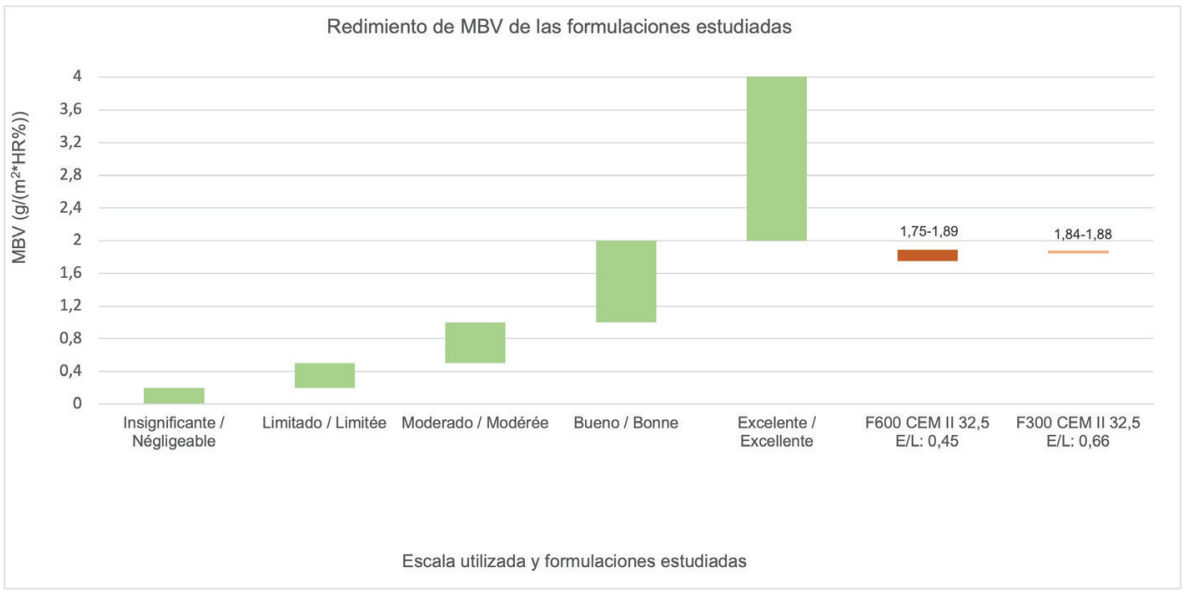

Figura 20. Rendimiento en MBV de los bio-concretos en estudio según escala de NORDTEST.

Cuadro 12. Valor de MBV para las formulaciones FFR y FCR.

\begin{tabular}{|c|c|c|c|c|c|c|c|}
\hline \multirow{2}{*}{ Ciclo } & \multirow{2}{*}{ Fase } & \multirow{2}{*}{$\Delta$ masa $(\mathrm{g})$} & \multirow{2}{*}{ Área expuesta $\left(\mathrm{m}^{2}\right)$} & \multicolumn{2}{|c|}{ Humedad relativa } & \multirow{2}{*}{\multicolumn{2}{|c|}{$\begin{array}{c}\text { MBV } \\
\left(\mathrm{g} /\left(\mathrm{m}^{2 *} \% \mathrm{HR}\right)\right)\end{array}$}} \\
\hline & & & & Alta & Baja & & \\
\hline \multicolumn{8}{|c|}{ FFR } \\
\hline \multirow{2}{*}{47} & Absorción & 1,67 & \multirow{6}{*}{0,02} & \multirow{6}{*}{75} & \multirow{6}{*}{33} & 1,98 & \multirow{2}{*}{$1,8 \varepsilon$} \\
\hline & Desorción & $-1,51$ & & & & 1,79 & \\
\hline \multirow{2}{*}{48} & Absorción & 1,66 & & & & 1,97 & \multirow{2}{*}{1,8} \\
\hline & Desorción & $-1,44$ & & & & 1,71 & \\
\hline \multirow{2}{*}{48} & Absorción & 1,60 & & & & 1,89 & \multirow{2}{*}{1,8} \\
\hline & Desorción & $-1,51$ & & & & 1,79 & \\
\hline \multicolumn{8}{|c|}{ FCR } \\
\hline \multirow[t]{2}{*}{27} & Absorción & 1,38 & \multirow{6}{*}{0,02} & \multirow{6}{*}{75} & \multirow{6}{*}{33} & 1,63 & \multirow{2}{*}{1,75} \\
\hline & Desorción & $-1,58$ & & & & 1,87 & \\
\hline \multirow[t]{2}{*}{28} & Absorción & 1,53 & & & & 1,81 & \multirow{2}{*}{$1,8 \varsigma$} \\
\hline & Desorción & $-1,67$ & & & & 1,98 & \\
\hline \multirow[t]{2}{*}{29} & Absorción & 1,36 & & & & 1,61 & \multirow{2}{*}{$1,7 €$} \\
\hline & Desorción & $-1,62$ & & & & 1,92 & \\
\hline
\end{tabular}


Del ensayo de transmisión de vapor de agua (Wetcup), se obtuvieron los siguientes resultados.

Cuadro 13. Valores promedio obtenidos del ensayo de Wetcup para especímenes estudiados de las formulaciones FFR y FCR.

\begin{tabular}{|c|c|c|c|c|c|}
\hline Formulación & $\begin{array}{c}\mathrm{G} \\
\mathrm{kg} / \mathrm{s}\end{array}$ & $\begin{array}{c}\mathrm{g} \\
\mathrm{kg} /\left(\mathrm{s}^{*} \mathrm{~m}^{2}\right)\end{array}$ & $\begin{array}{c}\mathrm{W} \\
\mathrm{kg} /\left(\mathrm{s}^{*} \mathrm{~m}^{2 *} \mathrm{~Pa}\right)\end{array}$ & $\begin{array}{c}\mathrm{Z} \\
\left(\mathrm{s}^{*} \mathrm{~m}^{2 *} \mathrm{~Pa}\right) / \mathrm{kg}\end{array}$ & $\begin{array}{c}\delta \\
\mathrm{kg} /\left(\mathrm{s}^{*} \mathrm{~m}^{*} \mathrm{~Pa}\right)\end{array}$ \\
\hline $\mathrm{FFR}$ & $-7,00 \mathrm{E}-08$ & $3,48 \mathrm{E}-06$ & $2,48 \mathrm{E}-10$ & $4,09 \mathrm{E}+09$ & $7,44 \mathrm{E}-11$ \\
\hline FCR & $-9,00 \mathrm{E}-09$ & $4,48 \mathrm{E}-07$ & $3,19 \mathrm{E}-10$ & $3,23 \mathrm{E}+09$ & $9,56 \mathrm{E}-12$ \\
\hline
\end{tabular}

Análisis económico

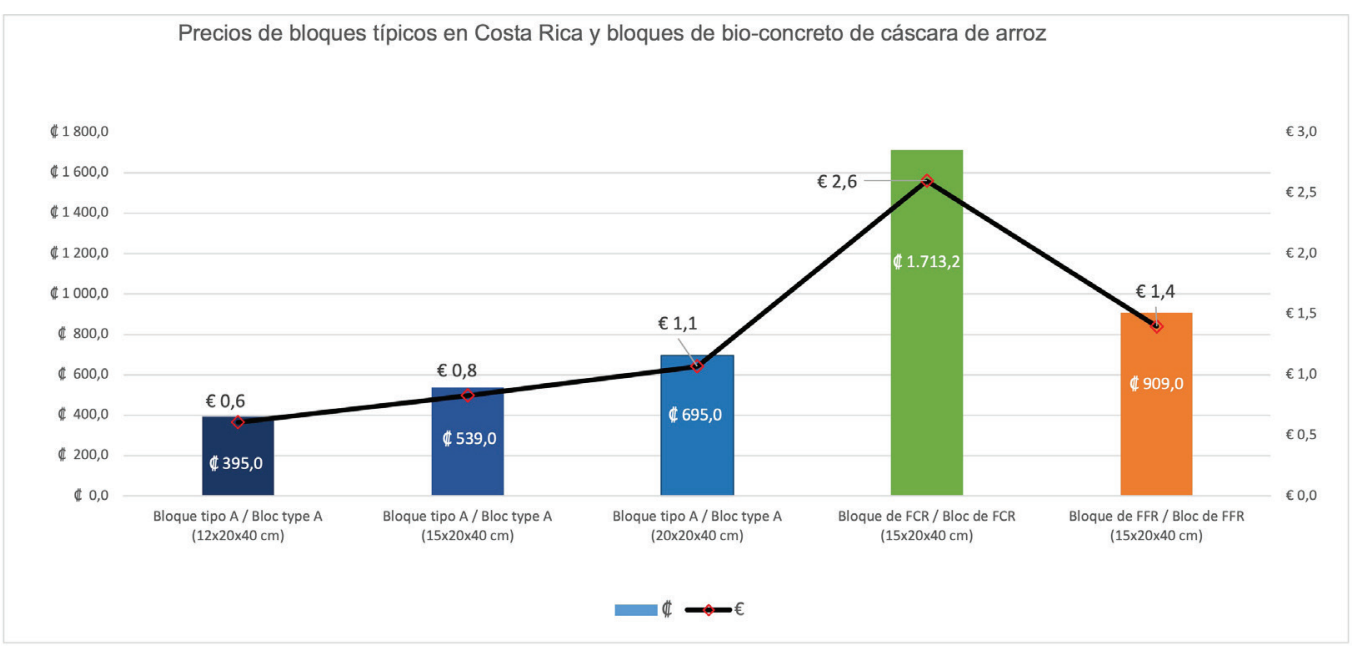

Figura 21. Costos de bloques típicos en Costa Rica tipo A según CSCR-10 y bloques de bio-concreto con cáscara de arroz (costos de elaboración en Costa Rica).

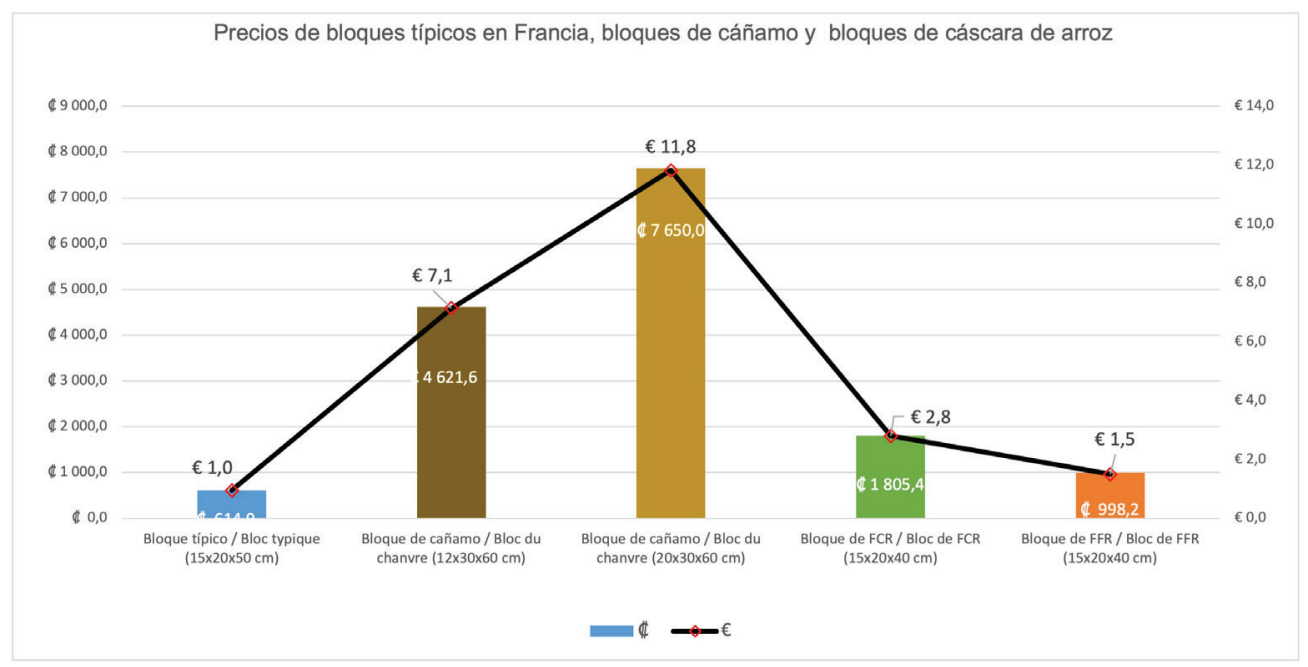

Figura 22. Costos de bloques típicos en Francia, bloques de cáñamo y bloques de bioconcreto con cáscara de arroz (costos de elaboración en Francia). 


\section{Análisis de Resultados}

\section{Caracterización de la cáscara de arroz}

La cáscara de arroz utilizada en este proyecto como único agregado de los bio-concretos elaborados, fue recibida en el laboratorio del ENTPE compactado, empacado en sacos y seco a temperatura ambiente.

Por lo que, al trabajar con este material y sin haberle dado un tratamiento físico ni químico previo a su caracterización física, se obtuvo que la densidad aparente no compactada es de $113 \mathrm{~kg} /$ $\mathrm{m}^{3}$, dato que no se aleja de los resultados obtenidos en estudios como el de [8] quien obtuvo una densidad no compactada de $143 \mathrm{~kg} / \mathrm{m}^{3}$, a comparación de un estudio más allegado a esta investigación en donde el material proviene de la misma fuente de distribución, el autor [7] reporta un valor de $110 \mathrm{~kg} / \mathrm{m}^{3}$.

La granulometría del material es poco dispersa entre los tamices utilizados, como se muestra en el cuadro 2 y figura 1, a partir del tamiz de $2,5 \mathrm{~mm}$ de apertura se tiene de manera acumulativa: de 1 a $6 \%$ del material el retenido en el tamiz 2,5 mm, de 80 a $84 \%$ del material retenido en el tamiz 1,25 mm, un 3,17\% retenido en el tamiz 1,00 mm, de 1,40 a 1,85\% retenido en el tamiz 0,63 mm y finalmente captado en la charola de 0,42 a 0,48\%. Por lo que, el tamaño más relevante para esta cáscara de arroz es de 1,25 mm dándole así el tamaño característico a este material.

El cuadro 3 muestra que en los primeros 30 minutos de contacto directo del material granular con el agua, se tiene una acelerada absorción, llegando a 120,51\% del peso de la cáscara de arroz. Como, de antemano se sabía que el tiempo de contacto de la cáscara de arroz con el agua y ligante, sería de 5 minutos en el proceso de mezclado, se calculó el porcentaje a este tiempo obteniendo una capacidad de absorción de agua de 104,01\% del peso de cáscara del arroz.

Importante obtener el dato de la capacidad de absorción de agua del material, debido a que en las mezclas de bio-concreto, se necesita proporcionar la cantidad correcta de agua para permitir la hidratación del ligante y además hidratar las fibras naturales. Con el fin, de que el agregado no quite agua necesaria para que se dé la reacción química entre agua/ligante que permite la generación de resistencia mecánica del material.

\section{Formulación de mezclas de bio-concreto}

Se obtuvo un bajo rendimiento de la resistencia a la compresión de las primeras 6 formulaciones estudiadas, estas no cumplían con la resistencia a la compresión mínima establecida en la norma INTE C89-2017, la única de estas formulaciones que presentó un rendimiento aceptable según los mínimos establecidos en el Protocolo de Prueba para la Medición del Rendimiento Umbral de Hormigones de Cáñamo, (0,3 MPa) para el caso de Francia, fue la F3 F250 CEM II $32,5 \mathrm{~A} / \mathrm{L}: 0,90$.

La decisión de utilizar solo cemento Portland tipo II como ligante en la segunda fase, se basó en el mejor rendimiento presentado en las formulaciones de la primera fase elaboradas con cemento y además que las dosificaciones de bio-concreto tenían el fin de utilizarse en la elaboración de bloques no estructurales. Por lo que, la obtención de altas resistencias a edades tempranas juega un papel importante en la posibilidad de desmoldar los especímenes con menores edades.

Al experimentar desmoldar los especímenes a temprana edad (1 día de elaboración) se podría comparar el desempeño mecánico ante compresión simple contra el desempeño presentado por especímenes desmoldados a mayor edad 21 días. Se obtuvo entonces, desde la formulación 
F8 hasta la F20 presentadas en el cuadro 5 que siempre había una variación significativa entre ambos desempeños, no siempre los especímenes desmoldados a mayor edad presentaban mayor resistencia ante la compresión, pero se tenía el factor de ingreso de los especímenes al horno en el proceso de curado por lo que se decidió analizar que pasaba si los especímenes no se ingresaban al horno y se desmoldaban con diferentes edades uno a 1 día de haberse elaborado y los otros dos a 28 días y someterlos al ensayo de compresión.

Con esto último, se verificaría si el desmolde a una edad temprana era el factor que afectaba en tener resistencias a la compresión tan variadas a comparación de las resistencias presentadas por los especímenes desmoldados a mayor edad, o bien si era el factor de haberlos ingresados al horno antes del ensayo. Esto último, se había realizado como sugerencia del Protocolo de Prueba para la Medición del Rendimiento Umbral de Hormigones de Cáñamo ya que no se tiene una normativa ni protocolo específico para el material en estudio se debía probar y descartar o aprobar los procedimientos de elaboración y ensayados de materiales similares como lo es el concreto hidráulico convencional y el concreto de cáñamo.

En el cuadro 6, se muestra entonces, con el ensayo de resistencia a los 28 días resultados menos dispersos, o bien se obtuvo el mismo desempeño mecánico entre los especímenes desmoldados con 1 día edad y los desmoldados a los 28 días para una misma formulación en estudio, por lo que el desmolde de los especímenes a edades tempranas no afecta directamente la resistencia de estos en tener menor desempeño con respecto a otro espécimen que se desmolda a edades mayores como 28 días. El desmolde de los especímenes a una edad temprana afecta directamente en la densidad final del espécimen, ya que en todos los casos siempre la densidad a los 28 días de los especímenes desmoldados con 1 día de elaboración fue menor que la presentada por los desmoldados con 21 o bien 28 días, el ingreso al horno afecto en tener un mayor rango de variación entre la densidad aparente final de los especímenes desmoldados a diferentes edades, al no ingresarlos al horno el rango de variación era menor pero siempre la densidad aparente final de los cilindros desmoldados a mayor edad independientemente de que se ingresaran al horno o no, fue mayor.

Se destaca, que no es recomendable desmoldar especímenes a edades tempranas (1 día) para formulaciones que tienen en su mezcla de $300 \mathrm{~kg}$ de ligante o menos por cada $113 \mathrm{~kg}$ de cáscara de arroz no compactada, es decir para las formulaciones F300 a menos, como se muestra en la figura 5, el espécimen de la formulación F280 se fracturó al desmoldarse a esa edad, el de la formulación F300 se le desprendió en la parte inferior material de la mezcla dejando un pequeño hueco en esta zona.

Las fallas típicas, para cada formulación se muestran en las figuras 6, 7, 8, se puede notar que entre menor la cantidad de cemento en la mezcla por ejemplo F8 F500 presenta una falla localizada en el tercio que menor compactación tuvo en la elaboración de los cilindros y conforme se va aumentado en cantidad de cemento en la mezcla por ejemplo las mezclas F16 a F19 las grietas empezaron por el tercio que menor compactación tuvo en la elaboración pero estas avanzaron a lo largo del cilindro, mostrando así una falla más frágil.

Finalmente las formulaciones escogidas para la elaboración de bloques no estructurales y otros elementos para el estudio de ensayos de resistencia mecánica como flexión y compresión simple fueron F12 F600 CEM II 32,5 A/L: 0,45 ya que a los 7 días de edad el promedio de resistencia a la compresión era de 4,1 MPa cumpliendo a esta edad con el mínimo establecido por INTE C89-2017 para Costa Rica de 4,41 MPa (a 28 días) se renombró a esta formulación FCR, para el caso de la normativa francesa se escogió la formulación F22 F300 CEM II 32,5 A/L: 0,66 a los 7 días de edad el promedio de resistencia a la compresión era de 0,3 MPa cumpliendo con el mínimo de resistencia para muros establecido en el Protocolo de Prueba para la Medición del Rendimiento Umbral de Hormigones de Cáñamo, renombrándola FFR. 
Elaboración de prismas, cilindros y bloques no estructurales a base de las formulaciones FCR y FFR para medición del rendimiento mecánico

Se replicó el ensayo de resistencia a la compresión, con el fin de constatar que al volver a producir cilindros y con los cuidados de no ingresarlos al horno y no desmoldarlos a menos de 7 días se tendrían resultados similares o los mismos a los obtenidos en el estudio de las formulaciones.

Para la formulación FCR el promedio de la resistencia a la compresión aumentó con respecto aumentaba la edad del espécimen, estos resultados fueron constantes con los obtenidos a los 7 y 28 días para los cilindros ensayados en la fase de estudio de formulaciones. Además, de un promedio de densidad aparente inicial de $1436 \mathrm{~kg} / \mathrm{m}^{3}$, permitiendo clasificar este bio-concreto como liviano según la clasificación dada en la norma ASTM C129 y así mismo INTE C89-2017. Por último, se reporta una resistencia a la compresión de 4,8 MPa y un módulo de elasticidad aparente de 529,9 MPa a los 28 días para esta formulación.

Para la formulación FFR, presentó un aumento de resistencia a la compresión respecto al aumento de edad, teniendo una congruencia con los datos obtenidos con la formulación F22 de la fase de estudio de formulaciones. Se cumple con el mínimo de 0,3 MPa a los 28 días demandado por el Protocolo de referencia y se clasifica como un bio-concreto liviano ya que su densidad aparente inicial es de $869 \mathrm{~kg} / \mathrm{m}^{3}$.

Por otro lado se obtuvo la resistencia a la flexión, en prismas elaborados con las formulaciones FCR y FFR, teniendo mayor desplazamiento y menor resistencia a la flexión en el prisma de FFR. No se utilizó ningún tipo de refuerzo para resistir la flexión (armadura de acero) así que el dato reportado es meramente de la resistencia aportada del bio-concreto teniendo una resistencia a la flexión de 2,75 MPa 70 días para la formulación FCR y 0,75 MPa a los 54 días para la formulación FCR.

Finalmente, el ensayo de resistencia a la compresión en bloques serían los resultados que dictarían si realmente el material cumplía con las solicitaciones mínimas demandas en las normas tomadas como base. Se obtuvo un promedio de resistencia a la compresión de 7,8 MPa a 78 días de edad en los bloques elaborados con FCR y para los bloques elaborados con la formulación FFR una resistencia de 1,5 MPa a 55 días de edad, ambas formulaciones cumplen satisfactoriamente los requisitos demandados.

Las fallas presentes en los bloques, se pueden clasificar con las mostradas en la norma ASTM C19, teniendo una falla tipo "Desprendimiento de Cara" para ambas formulaciones en donde es más visible en los especímenes de FCR ya que su alto contenido de cemento vuelve a los elemento más rígidos que los elaborados con FFR, aumentando con esto una falla más frágil para los elementos de FCR. Se puede observar estas fallas desde la figura 14 hasta la 19.

\section{Características Higrotérmicas de las formulaciones FCR y FFR}

Las características térmicas de ambas formulaciones se presentan en el cuadro 11, era importante conocer las capacidades de aislamiento térmico que presentarían estás formulaciones ya que, al construir con materiales que proporcionan mejor rendimiento en aislamiento térmico genera una menor demanda de la energía consumida en una edificación para tratar de controlar o mantener el confort térmico en los espacios de uso. Finalmente se calculó el tiempo de desfase térmico para ambas formulaciones con un ancho de $15 \mathrm{~cm}$ (ancho del bloque propuesto), con el fin de saber cuánto tiempo le tomaría a la temperatura exterior llegar hasta el interior de la edificación construida con este bio-concreto, teniendo entonces 8,94 horas para la formulación FFR y 8,35 horas para la formulación FCR. 
Se debe recordar que entre más cercano se el desfase térmico de un material a 12 horas mejor será como aislante térmico, suponiendo que a las 12 medio día se tiene la temperatura más alta en el día, esta temperatura teóricamente tardaría 8,94 horas en sentirse en el interior del inmueble, generando confort para el usuario puesto que la temperatura interior en el inmueble a las 21:34 horas del día será más cálida que la temperatura sentida en el exterior del inmueble, ya que en horas de la noche se tienen temperaturas más bajas.

Con la misma lógica entonces en horas del día cuando la temperatura es mayor, en el interior del inmueble se sentiría la temperatura registrada en el exterior hace 8,94 horas atrás. Esto sin necesidad de recurrir a sistemas de enfriamiento o calefacción.

Ahora bien, el material también muestra un buen rendimiento ante los cambio de humedad, ya que según la escala NORDTEST para el ensayo de MBV se puede clasificar el rendimiento de ambas formulaciones como "Bueno", siendo esto el segundo mejor puesto en la escala de calidad de amortiguamiento de humedad. Este resultado es respaldo con los obtenidos en el ensayo de Wetcup que mide la resistencia del material ante la penetración del vapor de agua $(Z)$, se muestra en el cuadro 13, que ambas formulaciones presentan una alta resistencia por $\mathrm{m}^{2}$ a que el material sea penetrado por humedad.

\section{Análisis económico}

Se tiene para la formulación FCR que no es viable económicamente producir bloques de bioconcreto ya que es $59,42 \%$ más caro que producir un bloque tipo A de 20x20x40 cm en Costa Rica, la brecha se da no solo en la parte económica sino también en el rendimiento mecánico ya que el bloque tipo A sí es estructural y permitido en todo tipo de construcciones en Costa Rica mientras que los de la formulación FCR al ser no estructurales su uso en construcciones se limita y son más caros.

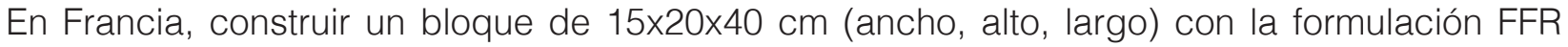
cuesta un 86,95\% más barato que un bloque de cáñamo de 20×30×60cm y 78,40\% más barato que un bloque de cáñamo de 12×30×60. Igualmente los costos presentados de los bloques de cáscara de arroz solo incluyen el costo de la materia prima, mientras que los bloques de cáñamo ya es el precio final como se ofrecen en una ferretería.

\section{Conclusiones}

1. La cáscara de arroz utilizada, proviene de la región francesa de Camarga, se le reporta un densidad aparente no compactada de $113 \mathrm{~kg} / \mathrm{m}^{3}$, tamaño granulométrico predominante de $1,25 \mathrm{~mm}$, un máximo de material fino retenido en la charola de $0,48 \%$ del peso tamizado y capacidad de absorción de agua a los 5 minutos de 104,01\% de su propio peso.

2. El desmolde a edades tempranas de cilindros de bio-concreto de cáscara de arroz garantiza tener menor densidad final en dichos cilindros con respecto a unos que se desmolden a mayor edad, no obstante este proceso no garantiza que el cilindro desmoldado a menor edad tendrá menor resistencia a la compresión con respecto al desmoldado con mayor edad.

3. La formulación escogida para la elaboración de los bloques no estructurales propuestos para Costa Rica fue la F12, renombrándola FCR F600 CEM II 32,5 A/L:0,45. Elegida por obtener a los 7 días una resistencia a la compresión de 4,1 MPa $\left(41,81 \mathrm{~kg} / \mathrm{cm}^{2}\right)$, lo cual cumple con el promedio de resistencia a la compresión demandada en la norma INTE C89- 2017 "Elementos de mampostería de concreto (bloques de concreto) para uso no estructural.". 
4. La formulación escogida para la elaboración de los bloques no estructurales propuestos para Francia fue la F22, renombrándola FFR F300 CEM II 32,5 A/L: 0,66. Elegida por obtener a los 7 días una resistencia a la compresión de 0,3 MPa $\left(3,06 \mathrm{~kg} / \mathrm{cm}^{2}\right)$, lo cual cumple con la resistencia a la compresión demandada en el "Protocolo de Prueba para la Medición del Rendimiento Umbral de Hormigones de Cáñamo".

5. Para la formulación FCR se reporta resistencia a la compresión en cilindros a los 28 de 4,8 MPa y módulo de elasticidad aparente de 529,9 MPa, resistencia a la flexión en prismas de 2,75 MPa asociado a un desplazamiento de 2,56 mm. Promedio de resistencia a la compresión en bloques no estructurales de 7,8 MPa a los 78 días.

6. Para la formulación FFR se reporta resistencia a la compresión en cilindros a los 28 de 0,6 MPa y módulo de elasticidad aparente de 61,5 MPa, resistencia a la flexión en prismas de 0,75 MPa asociado a un desplazamiento de 3,47 mm. Promedio de resistencia a la compresión en bloques no estructurales de 1,5 MPa a los 55 días

7. Los bloques ensayados a compresión simple de ambas formulaciones presentaron falla tipo "desprendimiento de cara" según ASTM C1314.

8. Las formulaciones FCR y FFR presentaron buen rendimiento como material aislante presentando así un tiempo de desfase térmico de 8,35 horas y 8,94 horas respectivamente.

9. No es viable económicamente producir los bloques con la formulación FCR por su alto contenido de cemento en la mezcla, sí es viable producir los bloques no estructurales con la formulación FFR para el caso de Francia pero para Costa Rica no puesto que estos no cumplen con la resistencia mínima demandada por las normas.

\section{Recomendaciones}

1. No se recomienda ingresar los cilindros de bio-concreto de cáscara de arroz al horno como parte del método de curado, debido a que esto puede generar pérdidas de la resistencia a la compresión de los especímenes.

2. No se recomienda desmoldar a menos de 7 días.

3. Realizar ensayo de resistencia a la compresión en especímenes tipo bloque a los 28 días y corroborar que a esta edad estos especímenes cumplen correctamente con los mínimos exigidos en las normativas.

4. Realizar un análisis de la respuesta sísmica que presentan los especímenes tipo bloques elaborados con la formulación FFR, ya que esta es económicamente viable de producir en ambos países, y sí el material presenta buen rendimiento ante fuerzas que simulen un sismo, generar una propuesta de estudio que regule a manera de norma la producción y aplicación de este producto dirigida a INTECO.

\section{Referencias}

[1] Lehne, J., \& Preston, F. (Junio de 2018). Chatham House Report. Obtenido de Making Concrete Change Innovation in Low-carbon Cement and Concrete: https://www.chathamhouse.org/sites/default/files/ publications/2018-06-13-making-concrete-change-cement-lehne-preston-final.pdf

[2] Global Cement and Concrete Association. (2019). Global context and quick definitions about concrete. London, United Kingdom.

[3] Federación Internacional del Cemento. (2018). Cifras de la industria cementera. Obtenido de Informe Estadístico 2018: http://ficem.org/dev/wp-content/uploads/2018/09/CIFRAS-DE-LA-INDUSTRIA-CEMENTERAMUNDIAL.pdf 
[4] Herrera Mejía, J., Osorio Saraz, J., \& Varón Aristizabal, F. (28 de Febrero de 2007). Comportamiento Mecánico del Concreto Reforzado con Fibras de Bagazo de Caña de Azúcar. Medellín, Colombia: Revista DYNA, Universidad Nacional de Colombia.

[5] Nguyen, T. (2010). Contribution à l'étude de la formulation et du procédé de fabrication d'éléments de construction en béton de chanvre. Génie civil. France: Université de Bretagne Sud.

[6] Molina Salas, E. (Enero de 2010). Evaluación del uso de la cascarilla de arroz en la fabricación de bloques de concreto. Cartago, Costa Rica: Tecnológico de Costa Rica.

[7] Mena Rodríguez, L. I. (2019). Análisis de las propiedades físicas de concretos modificados con fibras vegetales: cáscara de arroz. Cartago, Costa Rica: Tecnológico de Costa Rica - ENTPE.

[8] Serrano, T., Borrachero, V., Monzó, J., \& Paya, J. (2012). Morteros aligerados con cascarilla de arroz: Diseño de mezclas y evaluación de propiedades. DIALNET, 79, 128-136. 